\title{
Is there any relation between connective tissue growth factor and scar tissue in vesicoureteral reflux?
}

\author{
Nazlı Dilay Gültekin ${ }^{1}$, Meryem Benzer², Şebnem Tekin-Neijmann ${ }^{3}$ \\ Division of ${ }^{2}$ Pediatric Nephrology, ${ }^{1}$ Department of Pediatrics and ${ }^{3}$ Division of Biochemistry, Bakırköy Dr Sadi Konuk \\ Training and Research Hospital, Istanbul, Turkey. Email: dilayyenihan@hotmail.com \\ Received: 21st August 2017, Accepted: 2nd December 2017
}

SUMMARY: Gültekin ND, Benzer M, Tekin-Neijmann Ş. Is there any relation between connective tissue growth factor and scar tissue in vesicoureteral reflux. Turk J Pediatr 2019; 61: 71-78.

Vesicoureteral reflux (VUR) is the most common uropathy in childhood which leads to increased frequency of urinary tract infection (UTI) and renal scarring. Connective tissue growth factor (CTGF) plays an important role in the development of glomerular and tubulointerstitial fibrosis in progressive kidney diseases. The aim of this study was to investigate the relation between urinary CTGF and renal damage resulted from VUR. This cross sectional study included 70 patients with VUR and 62 healthy sex and age matched children. Urinary creatinine and CTGF (uCTGF) concentrations were analysed in all cases and CTGF to creatinine ratio were calculated. The records of radiologic evaluations of the patients including ultrasound, voiding cystouretrography and $99 \mathrm{~m}$-technetium dimercaptosuccinic acid (DMSA) scintigraphy were obtained retrospectively. The patient group was further divided into two groups according to the existence of renal cortical scarring in the DMSA scan. The study consisted of three groups; Group 1 (control group) 62 children, Group 2 (VUR positive, scar negative) 24 patient, Group 3 (VUR positive, scar positive) 46 patient (VUR+scar). The medians of uCTGF and uCTGF to creatinine ratio of the three groups were significantly different $(p<0.001)$. Pairwise group comparisons revealed that Group 1 had significantly lower uCTGF level and UCTGF/creatinine ratio, as compared to Groups 2 and 3 ( $p<0.001$ and $\mathrm{p}=0.002$, respectively). There was no statistically significant difference between Groups 2 and $3(p=0.052)$. uCTGF is significantly increased in children with VUR, independent on the presence of renal scarring. Increased uCTGF, even in the absence of the renal scarring, could be interpreted as development and a progression of glomerular and tubulointerstitial fibrosis in vesicoureteral reflux. Further experimental and clinical investigations are required to fully elucidate the mechanism of CTGF in vesicoureteral reflux.

Key words: connective tissue growth factor, vesicoureteral reflux, renal parenchymal scarring, reflux nephropathy, DMSA.

Vesicoureteral reflux (VUR) is the backward flow of urine from the bladder into the ureters and sometimes into the renal pelvis and calyces, due to ureterovesical junction defect. Primary vesicoureteral reflux is common among congenital urinary tract abnormalities. The development of renal parenchymal scarring (RPS) is associated with VUR. While renal parenchymal scarring is detected in $30-60 \%$ of children who are diagnosed with VUR for the first time, end-stage renal disease can be observed in $5-12 \%$ of them. ${ }^{1-8}$ The incidence of renal parenchymal scarring, which is also called reflux nephropathy, has been reported as $32 \%$ in Turkish children with chronic renal insufficiency. It has been shown that VURinduced renal parenchymal scarring increases the risk of developing hypertension and focal segmental glomerulosclerosis, and that if VUR is bilateral, it increases the risk of developing progressive renal failure. ${ }^{7-11}$

Although a vast amount of information is 
available about the diagnosis and treatment of VUR, questions still remain regarding how reflux leads to infection and renal damage. Furthermore, imaging is important in diagnosis and follow-up. Standard imaging modalities are renal ultrasound (USG), voiding cystourethrography (VCUG), and renal scintigraphy. Technetium-99m dimercaptosuccinic acid (DMSA) is a nuclear agent that shows most effectively the renal cortical tissue and the functional difference between the two kidneys. Noninvasive tests apart from such invasive and expensive imaging modalities are also needed in diagnosis and follow-up.

Renal fibrosis is the last common pathway for many kidney diseases that can progress to ESRD. As a consequence of inflammation and damage, humoral factors are secreted by infiltrating renal cells that stimulate the production of extracellular matrix molecules, which results in the disruption of normal function and integrity of the renal tissue. Connective tissue growth factor (CTGF), transforming growth factor- $\beta 1$ (TGF- $\beta 1$ ), platelet derived growth factor (PDGF), neutrophil gelatinase-associated lipocalin (NGAL), kidney injury molecule-1 (KIM-1), fibroblast growth factor (FGF), and bone morphogenetic protein (BMP7) are the most important mediators for fibrogenesis. ${ }^{12}$ For example, TGF- $\beta 1$ is the most potent fibrogenic factor in renal diseases and is the best indicator of renal damage.

CTGF is a member of the CCN family of secreted cysteine rich regulatory proteins. It stimulates renal fibroblast proliferation and extracellular matrix (ECM) synthesis. Three different cell types, including interstitial fibroblasts, mesenchymal cells and epithelial cells, have been shown to express CTGF mRNA. CTGF-positive cells are primarily myofibroblasts in the tubulointerstitial region, and it is synthesized together with $\alpha$-smooth muscle actin ( $\alpha$ SMA). CTGF is the major mediator independent of TGF- $\beta 1$ in fibrogenesis. ${ }^{13}$ In vitro studies have shown that CTGF participates in matrix synthesis and fibrosis. It was observed that CTGF mRNA expression was up-regulated in many diseases such as diabetic nephropathy and cardiomyopathy, fibrotic skin diseases, systemic sclerosis, biliary atresia, liver fibrosis, idiopathic pulmonary fibrosis, as well as non- diabetic acute or progressive glomerular and tubulointerstitial lesions. It has also been shown that CTGF plays a key role in the development and progression of diabetic renal fibrosis, and that urinary CTGF levels were also associated with the stage of diabetic nephropathy. ${ }^{14}$ Urinary CTGF levels were elevated especially in diabetic nephropathy. ${ }^{15}$

The purpose of this prospective study was to investigate the possibility of early detection of the relationship between urinary CTGF level and renal parenchymal scarring (RPS), which can develop secondary to reflux nephropathy without the need for other invasive tests.

\section{Material and Methods}

This study was conducted with 70 patients with vesicoureteral reflux and 62 healthy volunteers who were admitted to a pediatric nephrology outpatient clinic of a research and training hospital between January 2014 and December 2015. There were 49 girls and 21 boys in the patient group and 38 girls and 24 boys in the control group.

The patients were divided into 3 groups: Group 1 (control group) had no VUR or renal parenchymal scarring (62 children, mean age: $6.07 \pm 2.99$ years), Group 2 (only VUR) had VUR but no renal parenchymal scarring (24 children, mean age: $4.61 \pm 3.96$ years), Group 3 (VUR with renal parenchymal scarring) had both VUR and renal parenchymal scarring (46 children, mean age: $5.99 \pm 3.69$ years). The renal USG, VCUG and DMSA reports, which were obtained during routine examinations and tests of the patients in the study, were evaluated retrospectively. The diagnosis of VUR was evaluated according to the VCUG results and was staged between 1 and 5 in accordance with the International Study Classification (International Reflux Study Committee, 1981). ${ }^{16}$ VUR stages were added if VUR was bilateral (cumulative VUR score, CVS). The patients were divided into three groups according cumulative VUR score (TG) as follows: mild VUR (CVS $=1-2)$, moderate VUR (CVS $=3-6)$ and severe VUR (CVS $\geq$ $7)$. Renal scarring was diagnosed with DMSA. It was noted that DMSA scan was performed 3-6 months after urinary tract infection (UTI). According to the results of DMSA, the renal scarring was scored as follows: $0=$ normal, grade 
$1=$ one lesion (mild scar), grade $2=$ two lesions (moderate scar), grade 3 =diffuse renal scarring together with renal parenchymal damage (severe scar). ${ }^{17}$ Importantly, patients were excluded if they had a history of pyelonephritis during the period between withdrawal of DMSA and urine specimen collection. Therefore, it was accepted that the DMSA stages had not changed until the urine specimen collection. Those with a history of urinary tract infection, glomerulonephritis, urinary tract stone, major anomaly, and chronic disease were not included in the study.

The study protocol was approved by Institutional Ethics Committee. Parental consent was obtained from each case in the study after providing detailed information about the aims of the study.

Urine samples were collected from the patients for analysis of CTGF. They were asked to collect a midstream firstly in the morning on an empty stomach. The urine sample transferred into sterile containers was centrifuged at $3,000 \mathrm{rpm}$ for 5 minutes. The supernatant was stored at $-80^{\circ} \mathrm{C}$ until the time of analysis. After all samples were collected, they were analyzed with the ELISA (enzyme-linked immunosorbent assay) method based on the biotin double antibody sandwich technology for human CTGF assay (Catalog No: PHG0286,
Out Licensing, Life Technologies, 5791 Van Allen Way, Carlsbad, California 92008). Urine creatinine was measured by using the modified Jaffe method on an automated analyzer (AU 5830-Beckman Coulter, USA) and urine protein was measured on Cobas 6000 modular analyzer series (in c501 module, Roche, USA). Results were expressed as $\mathrm{pg} / \mathrm{ml}$, also expressed as $\mathrm{pg} / \mathrm{mg}$ creatinine, relative to creatinine (urinary protein normal: $<15 \mathrm{mg} / \mathrm{dl}$; trace: $15-30 \mathrm{mg} / \mathrm{dl}$; positive: $>30 \mathrm{mg} / \mathrm{dl}$ ).)

\section{Statistical analysis}

Statistical analysis was performed using the Statistical Software Package Program (Utah, USA), NCSS (Number Cruncher Statistical System) 2007. In order to evaluate the data, descriptive statistical methods (mean, standard deviation) were used. Moreover, the KruskalWallis test was used for intergroup comparisons, and the Dunn's multiple comparison test was used for subgroup comparisons. The MannWhitney $U$ test was performed to compare differences between two independent groups. The Chi-square and Fisher's exact test were used in order to compare qualitative data. The areas under the ROC curve for urinary CTGF/ creatinine $(\mathrm{pg} / \mathrm{mg}) 10^{3}$ and CTGF $(\mathrm{pg} / \mathrm{ml})$ were calculated. The sensitivity, specificity, positive predictive value (PPV), negative predictive value (NPV), likelihood ratio $(\mathrm{LR}+)$, and predictive

Table I. Comparison of Patient and Control Groups for Age, Gender, Height, Weight, BMI and Urinary Protein Positivity

\begin{tabular}{lcccc}
\hline & \multicolumn{4}{c}{ Study Groups } \\
\cline { 2 - 5 } Characteristics & $\begin{array}{c}\text { Controls } \\
(\mathrm{n}=62)\end{array}$ & $\begin{array}{c}\text { VUR with scar } \\
(\mathrm{n}=46)\end{array}$ & $\begin{array}{c}\text { VUR without scar } \\
(\mathrm{n}=24)\end{array}$ & $\mathrm{P}$ \\
\hline Age at sampling date (years) & $6.07 \pm 2.99$ & $5.99 \pm 3.69$ & $4.61 \pm 3.96$ & 0.112 \\
Gender, n (\%) & & & $4(16.7)$ & 0.139 \\
Male & $24(38.7)$ & $14(30.4)$ & $24(83.3)$ & \\
Female & $38(61.3)$ & $32(69.6)$ & $102.5 \pm 29.0$ & 0.097 \\
Height $(\mathrm{cm})$ & $115.4 \pm 19.2$ & $112.6 \pm 25.5$ & $20.99 \pm 15.43$ & 0.065 \\
Weight $(\mathrm{kg})$ & $24.95 \pm 13.03$ & $22.57 \pm 11.45$ & $17.73 \pm 2.81$ & 0.581 \\
BMI $\left(\mathrm{kg} / \mathrm{m}^{2}\right)$ & $18.07 \pm 7.23$ & $16.98 \pm 2.93$ & $19(79.2)$ & 0.344 \\
Urinary protein*, n (\%) & & & $5(20.8)$ & \\
Normal & $52(83.9)$ & $41(89.1)$ & $0(0.0)$ & \\
Trace & $9(14.5)$ & $3(6.5)$ & $2(4.5)$ & \\
Positive & $1(1.6)$ & &
\end{tabular}

BMI: body mass index, VUR: vesico-ureteral reflux.

*: normal: < $15 \mathrm{mg} / \mathrm{dl}$, trace: $15-30 \mathrm{mg} / \mathrm{dl}$, positive $>30 \mathrm{mg} / \mathrm{dl}$ 
Table II. Comparison of Patients According to Presence of Scar

\begin{tabular}{|c|c|c|c|}
\hline \multirow[b]{2}{*}{ Features } & \multicolumn{2}{|r|}{ Presence of scar } & \multirow[b]{2}{*}{$\mathrm{P}$} \\
\hline & VUR with scar & VUR without scar & \\
\hline Age at diagnosis (years) & $4.54 \pm 3.53$ & $2.97 \pm 3.5$ & 0.038 \\
\hline Cumulative VUR score & $1.83 \pm 0.64$ & $1.71 \pm 0.55$ & 0.345 \\
\hline Cumulative VUR score, n (\%) & & & 0.414 \\
\hline $1-2$ & $13(29.5)$ & $9(34.6)$ & \\
\hline $3-6$ & $25(56.8)$ & $16(61.5)$ & \\
\hline$\geq 7$ & $6(13.6)$ & $1(3.8)$ & \\
\hline Renal ultrasound, n (\%) & & & 0.336 \\
\hline Normal & $19(41.3)$ & $18(69.2)$ & \\
\hline Mild dilatation & $12(26.1)$ & $8(30.3)$ & \\
\hline Severe dilatation & $5(10.9)$ & 0 & \\
\hline Deformed & $10(21.7)$ & 0 & \\
\hline
\end{tabular}

VUR: vesicoureteral reflux

values were calculated in comparison to the control group. The results were evaluated in the confidence interval of $95 \%$, and significance was evaluated at the level of $\mathrm{p}<0.05$.

\section{Results}

\section{Characteristics of patients}

There were 70 children in the patient group (49 girls, $69.6 \%$; and 21 boys, $30.4 \%$ ), 62 children in the control group (38 girls, $61.3 \%$; and 24 boys, $38.7 \%$ ). At the date of sampling, there was no statistically significant difference between the groups in terms of age, height, weight, body mass index (BMI) values, gender distribution, and presence of protein in complete urine analysis ( $p>0.05)$ (Table I). The patient groups were compared in terms of the age at diagnosis, cumulative VUR score, and ultrasound findings according to presence of scar (Table II). The mean age at diagnosis of the VUR with scar group (4.54 \pm 3.53$)$ was found to be significantly higher than that of the VUR without scar group $(2.97 \pm 3.5)(p=0.038)$. There was no statistically significant difference in cumulative VUR score and ultrasound findings between groups. According to the DMSA results, of the patients in the VUR with scar group, $43.2 \%$ had a mild scar, $36.4 \%$ had a moderate scar and $20.5 \%$ had a severe scar.

Urinary creatinine, CTGF levels, protein/creatinine and CTGF/creatinine ratios in spot urine samples

There was a statistically significant difference between the groups in terms of urinary creatinine, CTGF levels and urinary protein/ creatinine and CTGF/creatinine ratios (Table III). The mean urinary creatinine level was found to be significantly lower in the VUR group than in the control group $(p=0.003)$. The mean urinary CTGF level and the mean urinary protein/creatinine and CTGF/creatinine ratios were found to be significantly lower in the control group compared to those in the VUR with scar and VUR without scar groups. There were no significant differences between the VUR with scar and VUR without scar groups in terms of these values.

Predictivity calculations of urinary CTGF/creatinine ratio and urinary CTGF level in VUR +scar and VUR groups compared to control group

The areas under the ROC curve for urinary CTGF/creatinine ratio and urinary CTGF level in the differential diagnosis of VUR with scar compared to the control group were found to be $0.646 \pm 0.054(0.539-0.753)$ and $0.722 \pm 0.048$ $(0.627-0.817)$, respectively. The areas under the ROC curve for urinary CTGF/creatinine ratio and urinary CTGF level in the differential diagnosis of VUR without scar compared to the control group were found to be $0.768 \pm 0.065$ $(0.640-0.895)$ and $0.737 \pm 0.064(0.612-$ 
Table III. Comparison of Groups in Terms of Urinary Creatinine and CTGF Levels and Urinary Protein/ creatinine and CTGF/creatinine Ratios

\begin{tabular}{lcccc}
\hline & \multicolumn{3}{c}{ Study Groups } & \\
\cline { 2 - 4 } Measurements & Controls & VUR with scar & VUR without scar & $\mathrm{p}$ \\
\hline $\begin{array}{l}\text { Urinary creatinine }(\mathrm{mg} / \mathrm{dl}) \\
\begin{array}{l}\text { Urinary protein/creatinine } \\
(\mathrm{mg} / \mathrm{mg})\end{array}\end{array}$ & $94.91 \pm 50.30$ & $78.1 \pm 49.44$ & $55.47 \pm 46.14$ & $<0.001$ \\
$\begin{array}{l}\text { Urinary CTGF/creatinine (pg/ } \\
\mathrm{mg}) \times 10^{3}\end{array}$ & $0.12 \pm 0.05$ & $0.18 \pm 0.14$ & $0.22 \pm 0.17$ & $<0.001$ \\
Urinary CTGF $(\mathrm{pg} / \mathrm{ml})$ & $439.45 \pm 100.12$ & $508.96 \pm 68.6$ & $521.2 \pm 95.17$ & $<0.001$ \\
\hline
\end{tabular}

CTGF: connective tissue growth factor; VUR: vesicoureteral reflux

0.862), respectively. There was no statistically significant difference between the areas under the ROC curve for urinary CTGF/creatinine ratio and urinary CTGF level in the differential diagnosis of VUR with scar and VUR without scar $(\mathrm{p}=0.152 ; \mathrm{p}=0.511)$.

Sensitivity, specificity, positive and negative predictive value and likelihood ratio of UCTGF/ creatinine ratio and uCTGF levels in the differential diagnosis are shown in Table IV.

\section{Discussion}

Vesicoureteral reflux (VUR) is among the most common congenital urological anomalies in children. Its incidence has been found to be about $1 \%$ in newborns but as high as $30-45 \%$ in children with urinary tract infection (UTI). ${ }^{18}$ It has been associated with an increased risk of UTI and renal scarring. It is diagnosed mostly after UTI. In a cohort of pediatric patients with UTI, including $68 \%$ infants, VUR was diagnosed in $33 \%$ of cases. ${ }^{19}$

Reflux nephropathy (RN) is defined as the formation of renal parenchymal scarring that is usually associated with UTI in patients with VUR. However, renal parenchymal scarring can be observed in the presence of UTI without VUR or in the presence of VUR without UTI. Children with VUR are more likely to develop pyelonephritis and renal scarring as compared to those with no VUR, and children with VUR grades III or higher are more likely to develop scarring than children with lower grades of VUR. ${ }^{6}$ The risk of renal scarring involving more than $25 \%$ of renal parenchyma is significantly higher in patients with grade III-IV (40\%) VUR as compared to those with grade I-II VUR $(14 \%)$ or no VUR $(6 \%) .{ }^{20}$ Therefore, children with VUR can lead to anxiety in both parents and physicians, because long-term follow-up and treatment are necessary in order to deal with its complications. Use of non-invasive tests and methods for diagnosis and followup of the disease would bring convenience to their families as well as physicians.

Connective tissue growth factor (CTGF) has an important role in embryogenesis, angiogenesis, wound healing, and tissue repair, especially in mesangial repair after kidney injury. Urinary CTGF levels (such as TGF $\beta$ ) may be useful in following renal diseases. Studies on CTGF have been conducted mainly in adults with diabetes. In a study conducted in 2003, Gilbert showed a relationship between uCTGF and the severity of diabetic nephropathy and emphasized the importance of this indicator. In the context of its known profibrotic effects, these findings suggest that CTGF contributes to chronic tubulointerstitial fibrosis accompanying proteinuric renal diseases. ${ }^{14}$ In our study, urinary CTGF level and urinary CTGF/ creatinine ratio was found to be increased in patients with VUR compared to the control group. However, urinary CTGF levels did not provide an additional contribution in the presence of RPS. In human studies, it has been reported that urinary CTGF expression shows a positive correlation with the severity of diabetic nephropathy and is a guide for progression of microalbuminuria to proteinuria. ${ }^{21}$ This suggests to us that proteinuria and urinary CTGF level may provide guidance for followups in terms of the development of RPS 
Table IV. Sensitivity, Specificity, Positive and Negative Predictive Value and Likelihood Ratio of uCTGF/ creatinine Ratio and uCTGF Levels in the Differential Diagnosis.

\begin{tabular}{|c|c|c|c|c|c|c|c|}
\hline & Parameters & Cut-off & $\begin{array}{c}\text { Sensitivity* } \\
(\%)\end{array}$ & $\begin{array}{c}\text { Specificity* } \\
(\%)\end{array}$ & $\begin{array}{c}\text { PPV* }^{*} \\
(\%)\end{array}$ & $\begin{array}{l}\mathrm{NPV}^{*} \\
(\%)\end{array}$ & $\mathrm{LR}^{*}$ \\
\hline \multirow[t]{2}{*}{$\begin{array}{l}\text { VUR without } \\
\text { scar vs. normal }\end{array}$} & $\begin{array}{l}\text { uCTGF/creatinine } \\
\quad(\mathrm{pg} / \mathrm{mg}) 10^{3}\end{array}$ & $>0.878$ & $\begin{array}{c}70.8 \\
(48.9-87.4)\end{array}$ & $\begin{array}{c}82.3 \\
(70.8-90.4)\end{array}$ & $\begin{array}{c}60.7 \\
(51.2-69.7)\end{array}$ & $\begin{array}{c}87.9 \\
(78.9-94.1)\end{array}$ & $\begin{array}{c}4.00 \\
(2.2-7.5)\end{array}$ \\
\hline & uCTGF (pg/ml) & $>495.46$ & $\begin{array}{c}70.8 \\
(48.9-87.4)\end{array}$ & $\begin{array}{c}72.6 \\
(60.4-82.9)\end{array}$ & $\begin{array}{c}50.0 \\
(41.7-58.3)\end{array}$ & $\begin{array}{c}86.5 \\
(77.5-93.6)\end{array}$ & $\begin{array}{c}2.58 \\
(1.6-42)\end{array}$ \\
\hline \multirow[t]{2}{*}{$\begin{array}{l}\text { VUR with scar } \\
\text { vs. normal }\end{array}$} & $\begin{array}{l}\text { uCTGF/creatinine } \\
\quad(\mathrm{pg} / \mathrm{mg}) 10^{3}\end{array}$ & $>0.91$ & $\begin{array}{c}45.7 \\
(33.6-58.1)\end{array}$ & $\begin{array}{c}85.5 \\
(75.1-93.1)\end{array}$ & $\begin{array}{c}70.0 \\
(62.5-77.9)\end{array}$ & $\begin{array}{c}67.9 \\
(59.9-75.1)\end{array}$ & $\begin{array}{c}3.15 \\
(1.8-5.6)\end{array}$ \\
\hline & uCTGF (pg/ml) & $>435$ & $\begin{array}{c}87.0 \\
(77.0-93.9)\end{array}$ & $\begin{array}{c}53.2 \\
(41.0-59.9)\end{array}$ & $\begin{array}{c}58.0 \\
(50.1-65.2)\end{array}$ & $\begin{array}{c}84.6 \\
(76.6-91.5)\end{array}$ & $\begin{array}{c}1.86 \\
(1.4-2.4)\end{array}$ \\
\hline
\end{tabular}

LR: likelihood ratio, NPV: negative predictive value, PPV: positive predictive value, uCTGF: urinary connective tissue growth factor, VUR: vesicoureteral reflux

$*$ : the values in parentheses represent $95 \%$ confidence intervals.

in patients with VUR. However, one of the limitations of our study was that there were deficiencies at follow-up of the urinary CTGF levels. Such an application would have been beyond the scope of this study, when taking into consideration the costs.

Proteinuria is one of the complications of $\mathrm{RN}$ that is more common in adult patients with VUR than in pediatric patients with VUR. Microalbuminuria has been found to be associated with renal scarring in $51 \%$ of pediatric patients in early stages of glomerular injury before progressive renal damage and renal failure developed. ${ }^{22}$ In our study, proteinuria levels were found to be lower in the control group than in the VUR with scar and VUR without scar groups. This supports that proteinuria begins before RPS develops and may be a good indicator of disease progression at follow-up.

In our study, of 44 patients with VUR and renal parenchymal scarring, $19(43.2 \%)$ had a mild scar, $16(36.4 \%)$ had a moderate scar and 9 $(20.5 \%)$ had a severe scar. Among the patients whom VUR stages were calculated according to VCUG results, $13.6 \%$ of the patients with RPS had high grade VUR (CVS $\geq 7$ ), whereas only $3.8 \%$ of the patients without RPS had high grade VUR. In accordance with the literature, this has confirmed the hypothesis that as VUR stage increases, the risk for renal scarring also increases. ${ }^{23}$

In our study, age at diagnosis was found to be significantly higher in the VUR with scar group than in the VUR without scar group. Increasing age at the diagnosis of VUR correlates with increasing incidence of renal scarring such that it is observed in $10 \%$ of preterm infants, $26 \%$ in children under 8 years, $47 \%$ in children older than 8 years and $94 \%$ in adults. ${ }^{24-25}$ In the study conducted by Hunziker et al. in 2014, according to the results of the assessment made in terms of VUR in siblings of patients with grade 3-5 VUR, they showed an increased risk for RPS in patients older than one year who had a history of UTI and high grade VUR. Therefore, lending evidence to the fact that early diagnosis of VUR is important for the development of RPS. ${ }^{23}$

In experimental diabetic nephropathy, overexpression of CTGF in glomeruli and tubulointerstitium increased glomerulosclerosis, tubulointerstitial fibrosis, and proteinuria. ${ }^{25-27}$ The urinary CTGF levels were normalized in these patients, in consistence with the improvement of tubular dysfunction with antiproteinuric measures. ${ }^{29}$ Therefore, monitoring of uCTGF values may gain importance as a noninvasive method in assessing renal function in situations such as VUR that can lead to chronic kidney failure.

In our study, uCTGF and uCTGF/creatinine values were significantly higher in the VURwith scar and VUR without scar groups compared to the control group. In line with our hypothesis, high levels of uCTGF in patients with VUR or VUR with RPS suggest that the fibrogenesis 
process persists even in the absence of RPS in patients with VUR. However, the lack of significant difference between the VUR and VUR with RPS groups may mean that UCTGF level is not a sufficient marker for pre-diagnosis of RPS or is not sensitive enough to show the presence of a small scar that is not yet detectable in DMSA. Furthermore, it is not known whether the increased levels of UCTGF, which is one of the factors responsible for mesangial repair after renal injury, prevent the progress of kidney disease. Since there is no other study on the relationship between the presence of VUR and uCTGF levels, a comparison could not be made to explain the possible link between this marker and fibrosis related to RN.

In conclusion; our study suggests that this noninvasive test may be useful in monitoring RPS associated with VUR but that there is a need for multicenter studies where a greater number of patients can be followed for standardization purposes.

\section{REFERENCES}

1. Smellie JM, Normand IC. Bacteriuria, reflux, and renal scarring. Arch Dis Child 1975; 50: 581-585.

2. Skoog SJ, Belman AB, Majd M. A nonsurgical approach to the management of primary vesicoureteral reflux. J Urol 1987; 138: 941-946.

3. Caione P, Ciofetta G, Collura G, Morano S, Capozza $\mathrm{N}$. Renal damage in vesico-ureteric reflux. BJU Int 2004; 93: 591-595.

4. Szlyk GR, Williams SB, Majd M, Belman AB, Rushton HG. Incidence of new renal parenchymal inflammatory changes following breakthrough urinary tract infection in patients with vesicoureteral reflux treated with antibiotic prophylaxis: Evaluation by $99 \mathrm{~m}$-technetium dimercapto-succinic acid renal scan. J Urol 2003; 170: 1566-1569.

5. Swerkersson S, Jodal U, Sixt R, Stokland E, Hansson S. Relationship among vesicoureteral reflux, urinary tract infection and renal damage in children. J Urol 2007; 178: 647-651.

6. Shaikh N, Ewing AL, Bhatnagar S, Hoberman A. Risk of renal scarring in children with a first urinary tract infection: A systematic review. Pediatrics 2010; 126: 1084-1091.

7. Jacobson SH, Eklöf O, Eriksson CG, Lins LE, Tidgren B, Winberg J. Development of hypertension and uraemia after pyelonephritis in childhood: 27 year follow up. BMJ 1989; 299: 703-706.

8. El-Khatib MT, Becker GJ, Kincaid-Smith PS. Reflux nephropathy and primary vesicoureteric reflux in adults. Q J Med 1990; 77: 1241-1253.
9. Claësson I, Jacobsson B, Jodal U, Winberg J Compensatory kidney growth in children with urinary tract infection and unilateral renal scarring: An epidemiologic study. Kidney Int 1981; 20: 759-764.

10. Lahdes-Vasama T, Niskanen K, Rönnholm K. Outcome of kidneys in patients treated for vesicoureteral reflux (VUR) during childhood. Nephrol Dial Transplant 2006; 21: 2491-2497.

11. Dillon MJ, Goonasekera CD. Reflux nephropathy. J Am Soc Nephrol 1998; 9: 2377-2383.

12. Bob F, Gluhovschi G. Processes of fibrogenesis in glomerular nephropathies. TMJ 2004; 54: 297-307.

13. Qi W, Chen X, Poronnik P, Pollock CA. Transforming growth factor-beta/connective tissue growth factor axis in the kidney. Int J Biochem Cell Biol 2008; 40: 9-13.

14. Gilbert RE, Akdeniz A, Weitz S, et al. Urinary connective tissue growth factor excretion in patients with type 1 diabetes and nephropathy. Diabetes Care 2003; 26: 2632-2636.

15. Nguyen T, Tarnow L, Andersen S, et al. Urinary connective tissue growth factor excretion correlates with clinical markers of renal disease in a large population of type 1 diabetic patients with diabetic nephropathy. Diabetes Care 2006; 29: 83-88.

16. Report of the International Reflux Study Committee. Medical versus surgical treatment of primary vesicoureteral reflux. Pediatrics 1981; 67: 392-400.

17. Imperiale A, Olianti C, Sestini S, et al. 123I-hippuran renal scintigraphy with evaluation of single-kidney clearance for predicting renal scarring after acute urinary tract infection: Comparison with $(99 \mathrm{~m}) \mathrm{Tc}-$ DMSA scanning. J Nucl Med 2003; 44: 1755-1760.

18. Willemsen J, Nijman RJ. Vesicoureteral reflux and videourodynamic studies: Results of a prospective study. Urology 2000; 55: 939-943.

19. Wu C-Y, Chiu P-C, Hsieh KS, Chui CL, Shih CH, Chiou YH. Childhood urinary tract infection: A clinical analysis of 597 cases. Acta Paediatr Taiwan 2004; 45: 328-333.

20. Gonzalez E, Papazyan J-P, Girardin E. Impact of vesicoureteral reflux on the size of renal lesions after an episode of acute pyelonephritis. J Urol 2005; 173: 571-574.

21. O'Seaghdha CM, Hwang SJ, Bhavsar NA, et al. Lower urinary connective tissue growth factor levels and incident CKD stage 3 in the general population. Am J Kidney Dis 2011; 57: 841-849.

22. Karlen J, Linne T, Wikstad I, Aperia A. Incidence of microalbuminuria in children with pyelonephritic scarring. Pediatr Nephrol 1996; 10: 705-708.

23. Hunziker M, Colhoun E, Puri P. Renal cortical abnormalities in siblings of index patients with vesicoureteral reflux. Pediatrics 2014; 133: 933-937.

24. Kincaid-Smith P, Becker G. Reflux nephropathy and chronic atrophic pyelonephritis: A review. J Infect Dis 1978; 138: 774-780.

25. Bourchier D, Abbott GD, Maling TM. Radiological abnormalities in infants with urinary tract infections. Arch Dis Child 1984; 59: 620-624. 
26. Yokoi H, Mukoyama M, Mori K, et al. Overexpression of connective tissue growth factor in podocytes worsens diabetic nephropathy in mice. Kidney Int 2008; 73 : 446-455.

27. Wang S, Denichilo M, Brubaker C, Hirschberg R. Connective tissue growth factor in tubulointerstitial injury of diabetic nephropathy. Kidney Int 2001; 60: 96-105.
28. Roestenberg P, van Nieuwenhoven FA, Joles JA, et al Temporal expression profile and distribution pattern indicate a role of connective tissue growth factor (CTGF/CCN-2) in diabetic nephropathy in mice. Am J Physiol Renal Physiol 2006; 290: 1344-1354.

29. Slagman M, Nguven T, Waanders F, et al. Effects of Antiproteinuric intervention on elevated connective tissue growth factor (CTGF/CCN-2) plasma and urine levels in nondiabetic nephropathy. Clin J Am Soc Nephrol 2011; 6: 1845-1850. 